\title{
Tumor sólido pseudopapilar de páncreas. Reporte de caso
}

\author{
Héctor Fabio Losada M. ${ }^{1}$, Diego Ardiles L. ${ }^{1}$, Pablo San Martín F. ${ }^{1}$ y Pablo Burgos V. ${ }^{1}$
}

'Departamento de

Cirugía, Traumatología y Anestesiología de la Universidad de la Frontera. Temuco, Chile.

Recibido el 12 de septiembre de 2019 y aceptado para pubicacion el 23 de diciembre de 2019

Correspondencia a: Dr. Héctor Fabio Losada M. hector.losada@ufrontera.c

\section{Pseudopapilar solid tumor of pancreas. Case Report}

Introduction: The pseudopapillary solid tumor of the pancreas is a rare entity that represents less than $1 \%$ of pancreatic neoplasms. It usually occurs in young women and only gives symptoms of a compressive nature once it has reached a large size. Given its uncertain biological behavior, the treatment is surgery. Case Report: We present the case of a 23-year-old woman with a 1-year history of epigastric pain evolution and weight loss. The imaging study demonstrated a solid-cystic heterogeneous mass dependent on the head of the pancreas of neoplasic appearance. A laparoscopic incisional biopsy was performed, the result of which was an undifferentiated malignant tumor, which was why the surgical resection was chosen. An open pancreatoduodenectomy was performed without incident with a favorable post operative. Histopathological and immunohistochemical analyzes were compatible with a solid pseudopapillary tumor of the pancreas. Key words: solid pseudopapillary tumor; pancreatic cancer; tumor.

\section{Resumen}

Introducción: El tumor sólido pseudopapilar del páncreas es una rara entidad que representa menos del $1 \%$ de las neoplasias pancreáticas. Suele presentarse en mujeres jóvenes y solo da síntomas de carácter compresivo una vez que alcanza un gran tamaño. Dado su comportamiento biológico incierto el tratamiento es la cirugía. Caso Clínico: Presentamos el caso de una mujer de 23 años con historia de 1 año de evolución de dolor epigástrico y baja de peso. El estudio imagenológico demostró una masa heterogénea sólida-quística dependiente de la cabeza del páncreas de aspecto neoplásico. Se realizó una biopsia incisional laparoscópica cuyo resultado fue de un tumor maligno indiferenciado, por lo que se optó por la resección quirúrgica. Se realizó una pancreatoduodenectomía abierta sin incidentes con un postoperatorio favorable. Los análisis histopatológicos e inmunohistoquímico fueron compatibles con un tumor sólido pseudopapilar de páncreas.

Palabras clave: tumor sólido seudopapilar; cáncer de páncreas; tumor.

\section{Introducción}

El tumor sólido pseudopapilar del páncreas o tumor de Gruber-Frantz, fue descrito por primera vez en $1959^{1,2}$. Si bien su prevalencia no se puede establecer con claridad, distintos autores apuntan a alrededor de un $1 \%$ de todas las neoplasias pancreáticas $^{2,5}$. Sus síntomas son inespecíficos y en general son secundarios al carácter compresivo de lesiones de un tamaño considerable. La resección quirúrgica completa con márgenes negativos ofrece la posibilidad de una excelente supervivencia a largo plazo ${ }^{2-5}$.

A continuación, presentamos un caso de una mujer de 23 años que presenta un tumor pseudopa- pilar dependiente de la cabeza del páncreas que se resuelve con pancreatoduodenectomía.

\section{Caso clínico}

Mujer de 23 años sin antecedentes mórbidos, con historia de 1 año de evolución caracterizado por dolor abdominal epigástrico persistente irradiado a dorso exacerbado con las comidas y baja de peso de $10 \mathrm{~kg}$ durante el mismo periodo. Su examen físico no presenta alteraciones. Se realizó ecografía abdominal la cual reveló colelitiasis múltiple, una vía biliar de calibre normal y una masa mal definida de 
la cabeza del páncreas. Se completó el estudio con una tomografía axial computada (Figuras 1 y 2) y resonancia nuclear magnética que evidenciaron una masa de aspecto neoplásico ubicada en la cabeza del páncreas heterogénea, de predominio sólido y algunas áreas quísticas, bien delimitada, aparentemente encapsulada, de 4,7 x 5,3 x 5,7 cm, con discreta captación de medio de contraste y acentuada restricción a la difusión del agua, que desplazaba hacia lateral y comprimía la segunda porción del duodeno, sin invasión de estructuras vecinas. Las pruebas de laboratorio solo demostraban discreta elevación de pruebas hepáticas (GGT 570, GOT 210, GPT 1.067). Los marcadores tumorales se encontraban dentro de límites normales (CA 125 5,5 U/ml, CA 19-9 7,2 U/ml, CEA < 0,5 ng/ml, AFP $1 \mathrm{ng} / \mathrm{ml})$. Se presenta caso en reunión multidisciplinaria donde se decide realizar una laparoscopía exploradora con toma de biopsia para descartar enfermedad irresecable. En el procedimiento se confirmó la presencia de una gran masa de aproximadamente $5 \mathrm{~cm}$ de diámetro, bien delimitada, dependiente de la cabeza de páncreas, que desplaza duodeno, no infiltrante, de carácter sólido con mínimo contenido hemático. Sin ascitis, ni metástasis hepáticas, ni carcinomatosis peritoneal. La paciente evoluciona favorablemente durante el postoperatorio y es dada de alta en espera del resultado de la biopsia. La biopsia diferida infor-

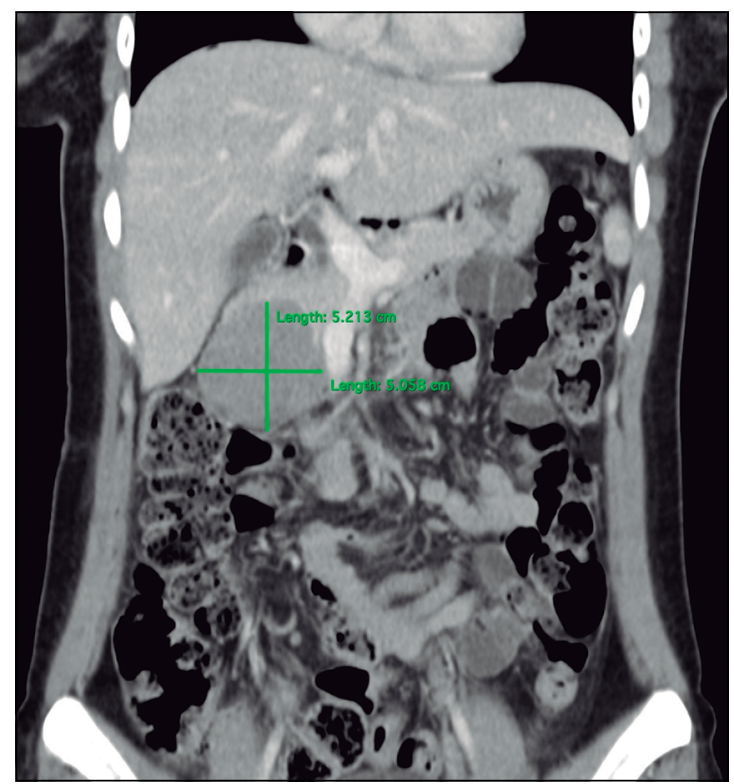

Figura 2. Tomografía computada con contraste en fase venosa corte coronal, Tumor bien delimitado dependiente de la cabeza del páncreas, de carácter heterogéneo en áreas quístico, de 5,2 x 5,1 cm. Eje venoso porto-mesentérico y arteria mesentérica superior sin signos de infiltración. ma tumor maligno poco diferenciado. Considerando los hallazgos descritos se decide realizar procedimiento de Whipple. Durante la cirugía se observó un tumor de aproximadamente $8 \mathrm{~cm}$ dependiente de la cabeza de páncreas que desplazaba parcialmente al duodeno, bien delimitado, sin diseminación macroscópica regional ni a distancia (Figura 3). La paciente evolucionó en forma favorable siendo dada de alta a los 7 días posteriores a la intervención. El estudio histopatológico no fue categóricamente concluyente (Figura 4), por lo que se complementó con análisis inmunohistoquímico revelando la expresión de

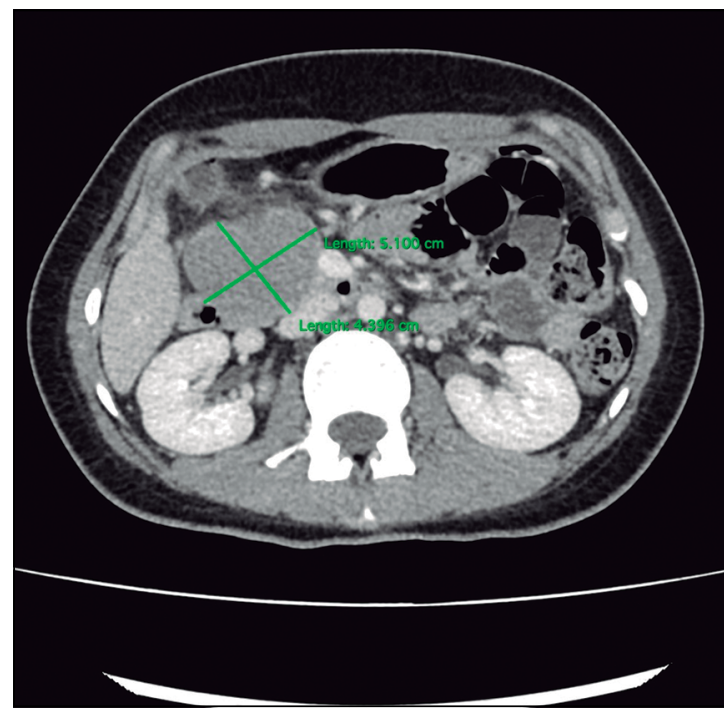

Figura 1. Tomografía computada con contraste en fase venosa corte axial, Tumor bien delimitado dependiente de la cabeza del páncreas, de carácter heterogéneo en áreas quístico, de 5,1 x 4,4 cm. Eje venoso porto-mesentérico y arteria mesentérica superior sin signos de infiltración. 


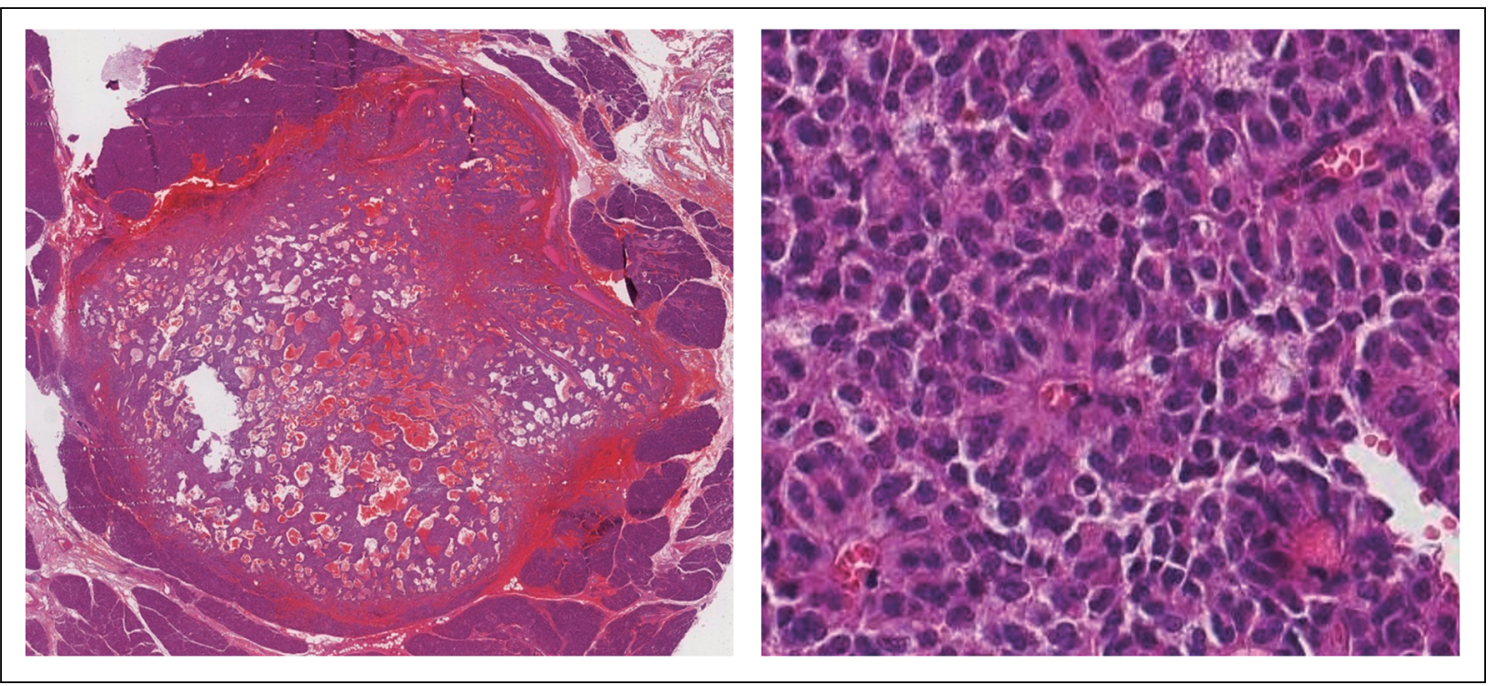

Figura 4. A: Lesión tumoral, de aspecto nodular, compuesta por áreas sólidas, pseudopapilares y hemorrágicas. El tumor se encuentra rodeado por una cápsula fibrosa que comprime y lo separa del parénquima pancreático. B: Se observan células neoplásicas monomorfas, poco cohesivas, que rodean tallos fibrovasculares, conformando estructuras pseudopapilares.

$\beta$-catenina, vimentina, CD10 y CD56, mientras que fue negativo para enolasa neuronal específica, sinaptofisina y cromogranina. Se confirmó la presencia de una neoplasia sólida pseudopapilar del páncreas. Los ganglios linfáticos y márgenes quirúrgicos fueron negativos y no presentaba invasión perineural ni linfovascular.

\section{Discusión}

El tumor de Franz es una rara entidad cuyo promedio de presentación es de 30 años, con relación de mujer a hombre de 10:11-4. Su localización más frecuente es en el cuerpo y cola del páncreas. A diferencia del resto de tumores pancreáticos, este tumor se caracteriza por su lento crecimiento y bajo grado de malignidad. Es de buen pronóstico, si bien su incidencia de transformación maligna es de $10 \%$, su recurrencia y sobrevida a 5 años es de 2 y $97 \%$ respectivamente ${ }^{4,5,9}$. Sin embargo, Yepuri recientemente estableció un drástico aumento de la recidiva, llegando hasta $23 \%$ en el periodo de 5 a 10 años, sugiriendo como factores de riesgo el sexo masculino, disección ganglionar positiva, presencia de invasión linfovascular y márgenes microscópicos positivos en la resección.

El origen celular es desconocido y podría incluir células ductales, acinares, endocrinas o células madre pluripotenciales. La mayoría presentan mutación del exón 3 de la $\beta$-catenina lo cual produce una acti- vación de la señal Wnt/ $\beta$-catenina mutación puntual de activación en el exón 3 del gen de la $\beta$-catenina (CTNNB1) $)^{2}$.

La presentación clínica inicial suele ser inespecífica. Debido a su crecimiento lento, a menudo provoca síntomas una vez alcanzado gran tamaño. El síntoma más frecuente es el dolor abdominal superior (11-67\%). Otros síntomas son distensión abdominal (16-27\%), masa abdominal palpable $(15 \%)$, náuseas y vómitos $(9 \%)$, pérdida de peso y ocasionalmente ictericia $(1 \%)^{3-5}$.

$\mathrm{Su}$ aspecto imagenológico típico consiste en una masa encapsulada con diversos componentes quísticos y sólidos causados por la degeneración hemorrágica; la calcificación y el leve realce intralesional también han sido descrito. Los tumores mayores a $3,5 \mathrm{~cm}$ presentan estas características clásicas, sin embargo, los tumores menores a $3 \mathrm{~cm}$ pueden presentarse solo como masas sólidas. La resonancia nuclear magnética permite identificar de mejor manera las áreas hemorrágicas entregando mayor información para el diagnóstico y permitiendo además definir la resecabilidad ${ }^{6,7}$.

La evaluación histológica muestra estructuras típicamente sólidas y pseudopapilares, vascularización intensiva o estructuras pseudoquísticas hemorrágicas en diversas proporciones. Los marcadores inmunohistoquímicos habitualmente utilizados en el TSP son vimentina, alfa-1-antitripsina, enolasa neuronal específica, CD10 y CD56 2,6,7.

En relación al tratamiento, en la actualidad la 
resección completa del tumor con margen R0 es el tratamiento de elección. El procedimiento quirúrgico depende de la localización y el tamaño del tumor, siendo la pancreatectomía distal con o sin esplenectomía para tumores del cuerpo y/o la cola del páncreas, y la pancreatoduodenectomía formal para tumores de la cabeza del páncreas. La resección completa es curativa con sobrevida a 5 años mayor a $95 \%$ de los pacientes con enfermedad limitada al páncreas. La disección ganglionar no es de regla dada la baja incidencia de metástasis linfática ${ }^{6-8}$. Cabe mencionar que el reciente trabajo de Yepuri reporta recurrencias aún después de los 5 años, sus- tentando la necesidad de mantener el seguimiento de estos pacientes al mediano y largo plazo.

\section{Responsabilidades éticas}

Protección de personas y animales. Los autores declaran que para esta investigación no se han realizado experimentos en seres humanos ni en animales.

Confidencialidad de los datos. Los autores declaran que en este artículo no aparecen datos de pacientes.

Conflictos de interés: no hay.

\section{Bibliografía}

1. Frantz VK. Atlas of Tumor Pathology, Section 7, Fascicles 27 and 28. Washington, DC, USA: Armed Forces Institute of Pathology, 1959:32-3.

2. Bardales RH, Centeno B, Mallery JS, Lai R, Pochapin M, Guiter G, et al. Endoscopic Ultrasound-Guided Fine Needle Aspiration Cytology diagnosis of solid pseudopapillary tumor of the pancreas. A rare neoplasm of elusive origin but characeristic cytomorphologic features. Am J Clinic Pathol. 2004;121:654-62.

3. Sperti C, Berselli M, Pasquali C, Pastorelli D, Pedrazzoli S. Aggressive behaviour of solid-pseudopapillary tumor of the pancreas in adults: a case report and review of the literature. World $\mathbf{J}$ Gastroenterol. 2008;14:960-5.

4. Zhang H, Wang W, Yu S, Xiao Y, Chen J. The prognosis and clinical characteristics of advanced (malignant) solid pseudopillary neoplasm of the pancreas. Tumour Biol. 2016;37:5347-53.

5. Papavramidis T, Papavramidis S. Solid pseudopapillary tumors of the pancreas: review of 718 patients reported in english literature, Journal of the American College of Surgeons 2005;200:965-72.

6. Jeng LB, Chen MF, Tang RP. Solid and papillary neoplasm of the pancreas.
Emphasis on surgical treatment. Arch Surg. 1993;128:433-6.

7. Tipton SG, Smyrk TC, Sarr MG, Thompson GB. Malignant potential of solid pseudopapillary neoplasm of the pancreas. Br J Surg. 2006;93:733-7.

8. Igbinosa O. Pseudopapillary tumor of the pancreas. An algorithmic approach. JOP 2011;12:262-5

9. Yepuri N, Naous R, Meier AH, Cooney RN, Kittur D, Are CA, et al. Systematic review and meta-analysis of predictors of recurrence in patients with Solid Pseudopapillary Tumors of the Pancreas. HPB (Oxford). 2020;22:12-9. doi: 10.1016/j.hpb.2019.06.005. Epub $2019 \mathrm{Jul} 23$. 